\title{
À propos d'une note critique tendancieuse de José Igartua
}

\section{Robert Armstrong}

Volume 40, numéro 2, automne 1986

URI : https://id.erudit.org/iderudit/304448ar

DOI : https://doi.org/10.7202/304448ar

Aller au sommaire du numéro

Éditeur(s)

Institut d'histoire de l'Amérique française

ISSN

0035-2357 (imprimé)

1492-1383 (numérique)

Découvrir la revue

Citer ce document

Armstrong, R. (1986). À propos d'une note critique tendancieuse de José Igartua. Revue d'histoire de l'Amérique française, 40(2), 273-277.

https://doi.org/10.7202/304448ar d'utilisation que vous pouvez consulter en ligne.

https://apropos.erudit.org/fr/usagers/politique-dutilisation/ 


\section{DÉBATS}

\section{A PROPOS D'UNE NOTE CRITIQUE TENDANCIEUSE DE JOSÉ IGARTUA}

Dans une note critique de cette revue, le professeur Igartua de l'Université du Québec à Montréal a signé une recension de mon livre, Structure and Change: An Economic History of Quebec ${ }^{1}$. Sa note critique donne un portrait inexact et tendancieux du livre. Cette mise au point soulève certaines inexactitudes commises par Monsieur Igartua:

1 - La théorie économique englobe un très grand éventail d'outillages. Tel qu'indiqué dès la première page de l'introduction de mon livre, aucune approche particulière, aucun modèle économique exclusif ne peut expliquer la croissance économique au Québec depuis le 16e siècle. Ainsi je n'ai pas adopté la théorie néo-classique comme cadre général d'interprétation. Au contraire, afin de jeter de la lumière sur le comportement économique, j'ai eu recours à divers outils de l'économiste contemporain, là où ils se sont avérés utiles.

2 - Dans le chapitre introductif j'ai suggéré que, dans sa formulation uni-sectorielle, la théorie néo-classique cadrait généralement avec les grandes lignes du commerce des fourrures à l'époque coloniale française, mais qu'elle laissait évidemment beaucoup de côté (p. xvii). Ceci dit, je ne suis pas revenu à ce modèle dans le reste du livre ${ }^{2}$. Dès le début de sa note critique, cependant, Monsieur Igartua s'obstine à ne pas vouloir faire la distinction entre la théorie néo-classique et l'ensemble de la pensée économique contemporaine.

3 - Contrairement à ce qu'affirme Monsieur Igartua, les concepts d'avantage comparé et du produit générateur ne font pas partie de la pensée néo-classique. L'avantage comparé est un concept développé par l'économiste David Ricardo bien avant le développement de la théorie néo-classique. L'approche du produit générateur fait partie d'une

1 José E. Igartua, «Le Québec de Pangloss: Structure and Change: An Economic History of Quebec", Revue d' histoire de l'Amérique française, 39,2 (automne 1985): 253-261.

${ }_{2}$ Dans un article publié au moment de la publication du livre, j'ai fait une critique du modèle néo-classique, et de son recours aux techniques marginalistes, quand il est appliqué à l'agriculture québécoise au 19e siècle. Voir Robert Armstrong, «The Efficiency of Quebec Farmers in 1851», Histoire sociale/Social History, 17,33 (May 1984): 149-163. 
contribution originale de Harold Innis à la conceptualisation de l'histoire économique québécoise et canadienne. Cette approche a été reprise et intégrée à de multiples cadres de référence ${ }^{3}$. Bien que je me sois servi des concepts d'avantage comparé et du produit générateur, j'ai pris soin de souligner les limitations de ces deux approches (pp. xvii, 6, 8-11, entre autres).

4 - En aucun moment, je n'ai fait usage du concept d'équilibre général. Voici que Monsieur Igartua sème à nouveau la confusion. Ce que j'ai essayé de faire dans le domaine de l'analyse des institutions, c'est d'indiquer comment les forces économiques ont influencé l'évolution du cadre institutionnel aussi bien que l'évolution de la société, des classes sociales, des mouvements sociaux et des idéologies. Je n'ai jamais suggéré que l'histoire de ces phénomènes se réduit à des causes ayant leur source uniquement dans la vie économique ou matérielle.

5 - Les rapports de force constituent un élément important dans la définition des structures économiques et, par extension, dans l'évolution de la croissance économique. Ils constituent ainsi un thème important des chapitres portant sur les structures institutionnelles (chapitres 4,5 et 9) ainsi que les chapitres portant sur le mouvement ouvrier (chapitres 12 et 15). Si l'histoire amérindienne - et les rapports de forces y associés - est absente du livre, c'est que je ne suis pas expert dans ce domaine et que, comme indiqué à la première page de la préface, j'ai décidé de laisser cet important sujet à d'autres auteurs.

6 - A aucun moment je n'ai décrit les Québécois d'antan comme étant des agents économiques libres et rationnels. Je crois que des modèles économiques postulant l'existence de tels agents peuvent servir à éclaircir certains aspects de l'histoire économique québécoise: c'est toute une différence. Pendant trop longtemps l'histoire économique bas-canadienne a eu recours aux postulats de l'irrationalité de l'habitant face aux choix économiques. Comme d'autres auteurs avant moi, tels Paquet et Wallot, j'ai interprété le comportement des Québécois sous l'angle de la rationalité comme le suggèrent les modèles en sciences sociales ${ }^{4}$. En s'opposant à l'utilisation de modèles économiques, Monsieur Igartua donne l'impression de vouloir s'opposer à toute analyse économique, pour ne pas dire toute analyse. C'est son privilège, mais je crois que la plupart des historiens ne le suivraient pas dans cette voie anti-intellectuelle.

7 - A la page 254 de la note critique, Monsieur Igartua affirme que les concepts d'avantage comparé et de produit générateur m'amènent

3 M. H. Watkins, «A Staple Theory of Economic Growth», Canadian Journal of Economics and Political Science, 24,2 (May 1963): 141-158, et «The Staple Theory Revisited», Journal of Canadian Studies, 12,5 (Winter 1977): 83-95.

4 Voir Peter D. McClelland, Causal Explanation and Model Building in History, Economics and the New Economic History (Ithaca, New York, Cornell University Press, 1975). 
«à proposer des interprétations qu'on ne peut qualifier autrement que de fantaisistes». A la suite de son propre résumé de ma pensée, il écrit:

Aucune de ces affirmations ne s'appuie sur quelque mesure que ce soit. La plupart contredisent ce que l'on sait de façon générale sur les modalités des premières implantations françaises en Amérique du Nord.

Or, c'est la critique de Monsieur Igartua qui ne s'appuie sur «quelque mesure que ce soit». En aucun moment il ne dit ce que «l'on sait de façon générale sur les modalités des premières implantations françaises en Amérique du Nord», pas plus que sa propre version des faits. Pour ma part, j'ai pris soin de dire que les considérations économiques ne furent certainement pas les seules variables affectant le taux d'immigration en Nouvelle-France, et peut-être même qu'elles n'avaient pas été les plus importantes (pp. 14-15). Cependant, elles ont certainement eu une influence et aident à comprendre, par exemple, les taux d'immigration différents en Nouvelle-France et en Nouvelle-Angleterre: un phénomène mesurable (voir tableau 2.1) que j'ai essayé d'expliquer de façon globale. Quoiqu'imparfait comme élément d'explication, l'analyse des exportations de la colonie (voir tableau 1.1) donne une indication empirique des forces qui sous-tendaient le flux d'immigrés vers le bassin Saint-Laurent.

De fait, j'ai amorcé le premier chapitre par une discussion de la croissance économique sans développement qui semble caractériser l'économie coloniale. De ce vieux thème, repris par les théoriciens du développement de sous-développement dans les années 1960 et 1970, Monsieur Igartua semble complètement ignorant. Il se contente de baptiser tout ce qu'il n'aime pas de «néo-classique».

8 - Je ne présente pas le peuplement de la Nouvelle-France comme «le fruit de décisions individuelles librement prises». Contrairement à ce que Monsieur Igartua dit à la page 255 de sa note critique, j'ai pris la peine de rappeler au lecteur que seulement un petit nombre d'immigrants français sont venus au Canada à leurs propres frais. Cela indique le peu d'attrait qu'avait la base de ressources au Canada à l'époque et la nécessité de subventions, pour ne pas parler de la coercition étatique. Mais en l'absence d'un effort massif de la part de l'État, le taux d'immigration négligeable reflétait les bénéfices économiques escomptés par les immigrants potentiels. Quant au choix de secteur économique une fois arrivé, l'immigrant moyen s'est orienté vers l'agriculture présumément afin de se nourrir et de se vêtir, et ceci malgré son manque d'expérience agraire. Il est certain que l'engagement dans une activité saisonnière, telle la traite des fourrures, représentait un choix risqué contre un salaire modeste.

9 - Dans sa discussion de la crise agricole au début du 19e siècle à la page 257, Monsieur Igartua perçoit une «contradiction essentielle 
entre l'interprétation de Ouellet et celle des tenants du modèle néoclassique». Ni Paquet et Wallot ni moi n'avons jamais fait référence au modèle néo-classique dans ce contexte. Nous avons tout simplement suggéré le postulat selon lequel les paysans se comportent en producteurs rationels. Le postulat de la rationalité des cultivateurs québécois est plus défendable que celui de l'irrationalité apparemment défendu par Messieurs Igartua et Ouellet. Il nous sert à suggérer d'autres explications compatibles avec les informations que nous possédons de l'époque. En l'absence d'une véritable crise économique avant les années 1830 - car on n'a pas démontré de façon concluante qu'il y en avait une avant - il est clair que le lien entre la chute des revenus agricoles et la montée du nationalisme ne peut se faire avant ces années-là. Voilà qui semble dépasser Monsieur Igartua.

10 - Pour ce qui est de l'émigration des Québécois au 19e siècle, Monsieur Igartua associe un modèle économique particulier avec ce qu'il appelle «le modèle néo-classique». Or, le modèle de Michael Todaro, que j'ai adapté aux circonstances, n'est aucunement lié à la pensée néo-classique. Bien plus, ayant lui-même constaté que j'ai soulevé des graves doutes quant à l'existence d'une crise agricole avant les années 1830 , deux paragraphes plus loin, à la page 258 , Monsieur Igartua me reproche de ne pas avoir abordé le sujet des ajustements dans les comportements démographiques à la «détérioration» du secteur agricole au cours de la première moitié du $19 \mathrm{e}$ siècle. Or, il n'y a rien à expliquer avant les années 1830 puisqu'il n'y a pas nécessairement «détérioration» de l'agriculture avant les années 1830 et l'émigration des années 1840 qui en résulte est un sujet que j'ai abordé explicitement dans les chapitres 5 et 6 (voir pp. 67, 85-86) ${ }^{5}$.

11 - Dans les chapitres 12 et 15 , j'ai analysé quelques-uns des changements dans la nature du travail, l'état des salaires et le niveau de vie des travailleurs qu'a provoqués l'industrialisation. Or, selon Monsieur Igartua:

Des transformations dans la nature du travail, de la perte de contrôle des travailleurs sur le procès de travail, de l'état des conditions de travail ou de niveau de vie des travailleurs, pas un mot. Pourtant, ces phénomènes, qui découlent eux aussi du jeu du marché, constituent le fondement de l'augmentation de la productivité qui caractérise la Révolution industrielle (p. 260).

Comme bien d'autres affirmations semblables éparpillées à travers sa note critique, cette dernière est lancée sans fondement.

5 A ce jour, nous n'avons pas d'indication empirique directe d'une émigration importante de Francophones hors Québec avant les années 1840. Cependant, Paquet et Smith ont exploré ce terrain par moyens indirects. Voir Gilles Paquet et Wayne R. Smith., «L'émigration des Canadiens français vers les États-Unis, 1790-1940: problématique et coups de sonde», l'Actualité économique, 59,3 (septembre 1983): 423-453. 
12 - Monsieur Igartua prétend que des pressions quelconques m'ont «forcé de conclure que les travailleurs québécois constituent, à cause de leur langue et de la mobilité réduite qui en découle, une main-d'oeuvre captive». Rien ne m'a forcé d'en arriver à cette conclusion si ce n'est les données empiriques. A la question suivante, à savoir «pourquoi ces facteurs culturels restreindraient-ils davantage la mobilité des Québécois francophones au $20 \mathrm{e}$ siècle qu'au $19 \mathrm{e}$ ?», la réponse aux pages 251 et 252 du livre est la suivante:

The rapid rate of urbanization that characterized Quebec's population in the second half of the nineteenth century continued until the 1930s. (See Table 15.3) People moved in response to higher incomes and greater job opportunities in the non-agricultural sectors of the economy. Income and job opportunity differentials between Quebec and neighbouring regions also induced many Quebecers to leave the province. The rate of emigration slowed after 1900, especially during the war years, but accelerated again in the 1920s. The abrupt decline in emigration to the United States during the years of the Great Depression resulted from a change in American immigration laws.

13 - Enfin, le danger de ne pas bien comprendre, c'est qu'on interprète mal et qu'on est porté à projeter beaucoup de son ignorance dans un propos critique. Monsieur Igartua ne sait pas très bien ce dont il parle quand il s'avance sur le terrain de l'économie la plus élémentaire. Qu'à cela ne tienne, il faut prendre la défense de l'histoire faible analytiquement contre l'histoire analytiquement rigoureuse. Il ressort donc de la note critique de Monsieur Igartua une grande confusion.

Conseil de la radiodiffusion et des télécommunications canadiennes Ottawa 